\title{
What have we learned from animal models of ventilator-induced lung injury?
}

\author{
Patricia Rieken Macedo Rocco ${ }^{1 *}$ (ID and John J. Marini ${ }^{2}$
}

(c) 2020 Springer-Verlag GmbH Germany, part of Springer Nature

Despite its obvious benefits, mechanical ventilation can inflict pulmonary structural damage [1] and destabilize hemodynamics [2]. Deleterious effects of mechanical ventilation-collectively known as ventilator-induced lung injury (VILI)-include inflammatory infiltration and vascular permeability, hyaline membrane formation, and pulmonary edema [3]. Interactive mechanical forces prompt biophysical, biochemical, and biomolecular alterations that ultimately lead to VILI [4]. Repeated nonphysiological stretching of lung tissue can release inflammatory mediators, alter gene expression, and either upregulate or downregulate synthesis of several extracellular matrix proteins [5]. Therefore, understanding the physiological and biological consequences of mechanical ventilation and becoming familiar with logical clinical strategies intended to prevent and minimize lung damage are important clinical goals. These cannot be studied directly at the bedside for obvious ethical and logistical reasons. Yet, over the past two decades, the experimental laboratory has provided the needed guidance to develop approaches and equipment modifications essential to VILI prevention.

Animal models continue to elucidate mechanisms underlying the complex pathophysiology of VILI, aiding immeasurably in developing preventative and therapeutic approaches. Important factors upon which to focus when selecting an animal model of VILI include availability, cost, public opinion regarding the conduct of animal research (e.g., feelings toward dogs or primates compared to rodents), the number of animals that should be used,

\footnotetext{
*Correspondence: prmrocco@gmail.com

${ }^{1}$ Laboratory of Pulmonary Investigation, Carlos Chagas Filho Institute of Biophysics, Federal University of Rio de Janeiro Centro de Ciências da Saúde, Av. Carlos Chagas Filho, 373, Bloco G-014, Ilha do Fundão, Rio de Janeiro, RJ 21941-902, Brazil

Full author information is available at the end of the article
}

and the availability of species-specific reagents to measure inflammatory mediators, receptors, or other proteins [6]. Different species are best suited to different study objectives; if survival is the primary outcome of interest, rodents provide an ideal option, since large numbers are required. Mouse models of injury have specific reagents available and can be genetically modified. Conversely, if invasive monitoring or multiple blood samples are required, large animals share similarities of chest anatomy and mechanical properties with humans and provide easier anatomic access and adequate blood volumes. So far, no available animal model perfectly mimics certain key aspects of human VILI, limiting the breadth of our understanding and impeding rapid extrapolation of findings to the clinical setting. For example, the intensity and evolution of VILI differ between experimental and human studies. Importantly, most animal models do not receive therapies, such as fluid intake, which influence outcomes in humans. Despite such limitations, precise regulation of the components of the mechanical stimulus and the monitoring of their effects in animal models has enabled acquisition of detailed mechanistic knowledge of undeniable clinical import [7].

\section{Recent laboratory discoveries Mechanical forces}

The experimental setting allows investigation of purely mechanical (wounding and signaling) stimuli on tissue responses. Furthermore, mechanical forces can be explored over wide ranges and under conditions that would be neither feasible nor ethical in human patients. Some of these contributors, e.g. PEEP [8], may have protective or damaging influence, depending on range and biological substrate. Ventilation patterns have been explored both in small and large animals, which differ in their susceptibility to mechanical stresses and strain depending on size, age, and pre-existing inflammatory

\section{Springer}


state. Transpulmonary pressures, differing regional tidal excursions, and associated injury potentials have been measured in large animals, confirming the importance of local chest wall properties to VILI hazard [9]. The benefit of prone positioning for leveling the distribution of transpulmonary pressures and reducing gravitationally dependent VILI has been demonstrated clearly in healthy large animals and in models of preinjury [10].

Recent work has made clear that surpassing minimum thresholds for tidal stress and strain may be required to initiating lung injury in healthy as well as pre-injured lungs $[4,11]$ and that both static and dynamic stresses impact the actual strains experienced at the cellular level [7]. Micromechanical amplifiers of stress include geometric heterogeneity within the parenchyma, differential viscoelastance and rates of parenchymal expansion, and progressive loading due to dropout of weaker stress-bearing matrix fibrils as tidal cycling proceeds (If unrelieved or interrupted, the latter process may eventuate catastrophic alveolar barrier breakdown.). The propagation of parenchymal injury and the inflammatory process can now be traced by advanced imaging techniques. Accentuated surface forces develop at the boundary of closed and open lung units, inciting inflammation. Such phenomena have been especially well demonstrated during repeated tidal opening and closing of small airways [4]. In that setting, the importance of surfactant depletion and shearing related to fluid movements have been experimentally verified, as has the protective effect of PEEP.
A series of ongoing studies have demonstrated that each individual element of tidal energy and power, even PEEP, contributes to tissue damage (albeit with different potency and effect) if it is applied above the aforementioned injury threshold $[8,11]$. With that proviso, total power-the product of minute ventilation with the sum of flow resistive, tidal expansive, and baseline pressuresis a single integrating variable with the potential to track VILI risk [11, 12]. Predictably, duration of a damaging ventilation pattern influences eventual injury expression. Interrupting the monotony of passive cycling with "noisy" or biologically variable ventilation appears to confer some protection [13].

\section{Nonmechanical forces}

Nonmechanical factors condition the injury resulting from an adverse ventilation pattern. These include vascular pressures and flows, body temperature, $\mathrm{PaO}_{2}, \mathrm{PCO}_{2}$ and tissue $\mathrm{pH}$ (Fig. 1). Exactly similar patterns of inflation may cause radically different severities and types of VILI expression depending on the environment in which they occur.

The injury process is an ongoing competition between injurious with adaptive and reparative forces. The latter have been relatively little studied and are currently under investigation. At the cellular level, confocal microscopy has demonstrated lipid trafficking to the cellular surface that attenuates modest degrees of boundary stretch, deformation, and signaling. Very recent work in animal experiments has also shown that recruiting pressures

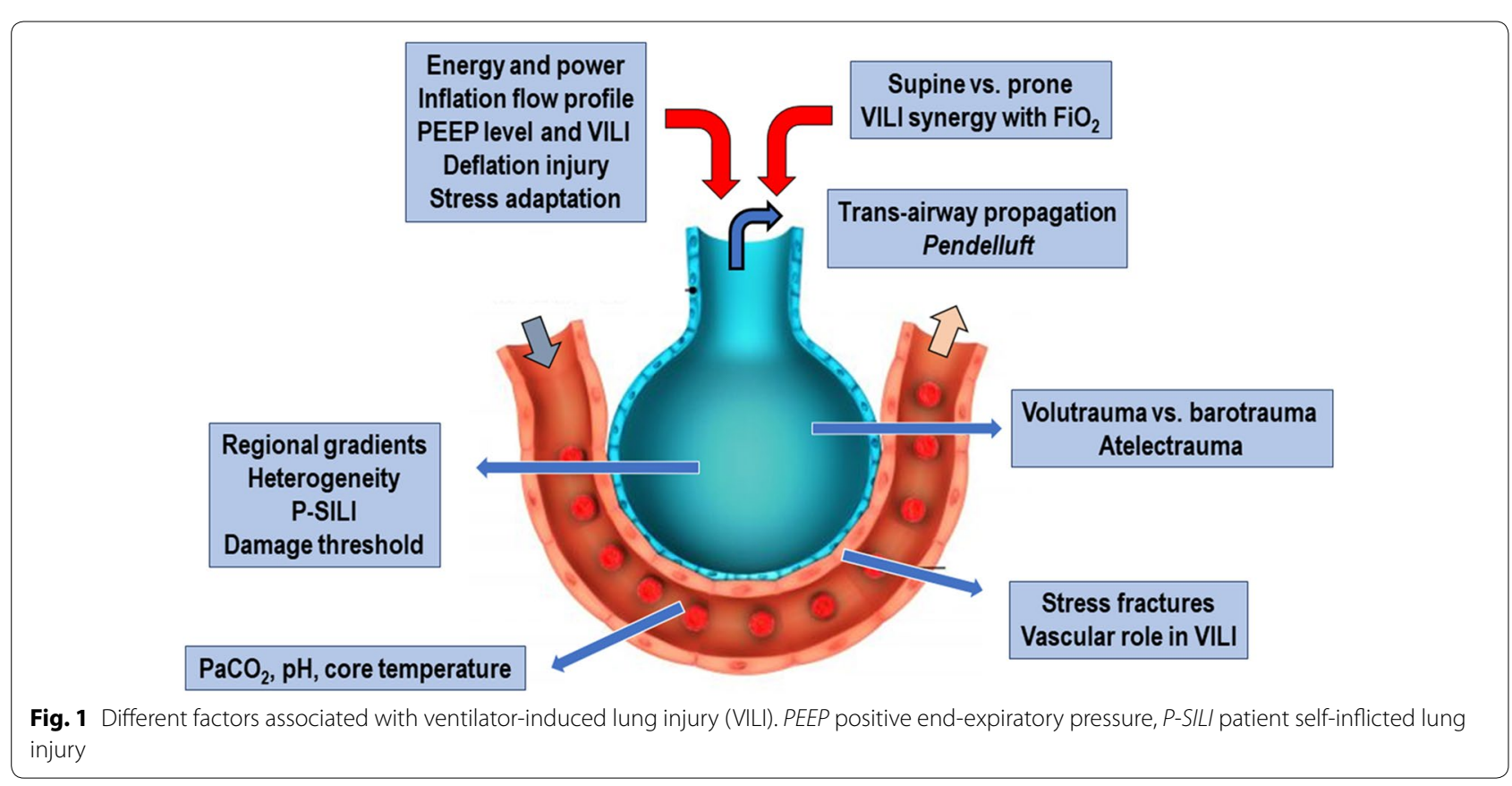


reached gradually over longer intervals are better tolerated than those applied abruptly [14]. Similarly, potentially injurious patterns of tidal stress may cause less damage if gradually escalated over time [15]. Recent experiments conducted in large animals have opened a new area of inquiry by demonstrating that slowing the initial release of ventilating pressures may attenuate the risk of VILI and improve hemodynamic tolerance [16].

\section{Future research directions}

Numerous questions regarding the safety of mechanical ventilation remain to be explored in animal models. Foremost among these are projects to refine the components of power as VILI risk factors, define time-dependent thresholds for mechanical injury, explore expiratory events in VILI causation, and precisely determine the codependence of clinician-modifiable mechanical and non-mechanical influences (such as fluid loading) on VILI. The contributions of the vascular side of the alveolar-capillary barrier to VILI requires additional exploration, as emphasized by the unique challenges presented by the vaso-centricity of COVID-19 pneumonia. Easing the abruptness of pattern transitions, as well as modifying the flow patterns of both inspiration and expiration, hold considerable potential to mitigate the risk of VILI. Multiple unresolved issues remain regarding how best to differentiate acute lung injury from the additive insult of an adverse ventilation pattern, whether spontaneous or fully controlled. How best to track the emergence and progress of iatrogenic ventilation patterns is eminently worth investigating, as are the healing processes of adaptation and repair.

\section{Author details \\ ${ }^{1}$ Laboratory of Pulmonary Investigation, Carlos Chagas Filho Institute of Bio- physics, Federal University of Rio de Janeiro Centro de Ciências da Saúde, Av. Carlos Chagas Filho, 373, Bloco G-014, Ilha do Fundão, Rio de Janeiro, RJ 21941-902, Brazil. ${ }^{2}$ University of Minnesota and Regions Hospital, Regions Hospital MS 11213 B, 640 Jackson St., St. Paul 55101, MN, USA.}

\section{Acknowledgements}

Supported by the Brazilian Council for Scientific and Technological Development (CNPq), Carlos Chagas Filho Rio de Janeiro State Research Foundation (FAPERJ), and Coordination for the Improvement of Higher Education Personnel (CAPES). We express our gratitude to Mr. Filippe Vasconcellos for his assistance in editing the manuscript,

\section{Funding}

This work was funded by Conselho Nacional de Desenvolvimento Científico e Tecnológico (Grant number: 421067/2016-0), Fundação Carlos Chagas Filho de Amparo à Pesquisa do Estado do Rio de Janeiro (Grant number: E-26/210.910/2016), and Coordenação de Aperfeiçoamento de Pessoal de Nível Superior (Grant number: 23038.005800/2017-41).

\section{Compliance with ethical standards}

\section{Conflicts of interest}

The authors have no conflict of interest to disclose.

\section{Publisher's Note}

Springer Nature remains neutral with regard to jurisdictional claims in published maps and institutional affiliations.

Received: 29 April 2020 Accepted: 26 May 2020

Published online: 4 June 2020

\section{References}

1. Grune J, Tabuchi A, Kuebler WM (2019) Alveolar dynamics during mechanical ventilation in the healthy and injured lung. Intensive Care Med Exp 25:34

2. Katira BH, Giesinger RE, Engelberts D, Zabini D, Kornecki A, Otulakowski G, Yoshida T, Kuebler WM, McNamara PJ, Connelly KA, Kavanagh BP (2017) Adverse heart-lung interactions in ventilator-induced lung injury. Am J Respir Crit Care Med 196:1411-1421

3. Cruz FF, Ball L, Rocco PRM, Pelosi P (2018) Ventilator-induced lung injury during controlled ventilation in patients with acute respiratory distress syndrome: less is probably better. Expert Rev Respir Med 12:403-414

4. Marini JJ, Rocco PRM, Gattinoni L (2020) Static and dynamic contributors to VILI in clinical practice: pressure, energy, and power. Am J Respir Crit Care Med 201:767-774

5. Pelosi P, Rocco PR (2008) Effects of mechanical ventilation on the extracellular matrix. Intensive Care Med 34:631-639

6. Rocco PRM, Nieman GF (2016) ARDS: what experimental models have taught us. Intensive Care Med 42:806-810

7. Santos RS, Maia LA, Oliveira MV, Santos CL, Moraes L, Pinto EF, Samary CDS, Machado JA, Carvalho AC, Fernandes MVS, Martins V, Capelozzi VL, Morales MM, Koch T, Gama de Abreu M, Pelosi P, Silva PL, Rocco PRM (2018) Biologic impact of mechanical power at high and low tidal volumes in experimental mild acute respiratory distress syndrome. Anesthesiology 128:1193-1206

8. Collino F, Rapetti F, Vasques F, Maiolo G, Tonetti T, Romitti F, Niewenhuys J, Behnemann T, Camporota L, Hahn G, Reupke V, Holke K, Herrmann P, Duscio E, Cipulli F, Moerer O, Marini JJ, Quintel M, Gattinoni L (2019) Positive end-expiratory pressure and mechanical power. Anesthesiology 130:119-130

9. Cortes-Puentes GA, Keenan JC, Adams AB, Parker ED, Dries DJ, Marini JJ (2015) Impact of chest wall modifications and lung injury on the correspondence between airway and transpulmonary driving pressures. Crit Care Med 43:e287-295

10. Valenza F, Guglielmi M, Maffioletti M, Tedesco C, Maccagni P, Fossali T, Aletti G, Porro GA, Irace M, Carlesso E, Carboni N, Lazzerini M, Gattinoni $L$ (2005) Prone position delays the progression of ventilator-induced lung injury in rats: does lung strain distribution play a role? Crit Care Med 33:361-367

11. Marini JJ, Rocco PRM (2020) Which component of mechanical power is most important in causing VILI? Crit Care 24:39

12. Santos RS, Maia LA, Oliveira MV, Santos CL, Moraes L, Pinto EF, Samary CDS, Machado JA, Carvalho AC, Fernandes MVS, Martins V, Capelozzi VL, Morales MM, Koch T, Gama de Abreu M, Pelosi P, Silva PL, Rocco PRM (2018) Biologic impact of mechanical power at high and low tidal volumes in experimental mild acute respiratory distress syndrome. Anesthesiology 128:1193-1206

13. Wittenstein J, Scharffenberg M, Braune A, Huhle R, Bluth T, Herzog M, Güldner A, Ball L, Simonassi F, Zeidler-Rentzsch I, Vidal Melo MF, Koch T, Rocco PRM, Pelosi P, Kotzerke J, Gama de Abreu M, Kiss T (2020) Effects of variable versus nonvariable controlled mechanical ventilation on pulmonary inflammation in experimental acute respiratory distress syndrome in pigs. Br J Anaesth. https://doi.org/10.1016/j. bja.2019.12.040

14. Santos RS, Moraes L, Samary CS, Santos CL, Ramos MB, Vasconcellos AP, Horta LF, Morales MM, Capelozzi VL, Garcia CS, Marini JJ, Gama de Abreu M, Pelosi P, Silva PL, Rocco PR (2016) Fast versus slow recruitment maneuver at different degrees of acute lung inflammation induced by experimental sepsis. Anesth Analg 122:1089-1100

15. Felix NS, Samary CS, Cruz FF, Rocha NN, Fernandes MVS, Machado JA, Bose-Madureira RL, Capelozzi VL, Pelosi P, Silva PL, Marini JJ, Rocco PRM 
(2019) Gradually increasing tidal volume may mitigate experimental lung injury in rats. Anesthesiology 130:767-777

16. Del Sorbo L, Goligher EC, McAuley DF, Rubenfeld GD, Brochard LJ, Gattinoni L, Slutsky AS, Fan E (2017) Mechanical ventilation in adults with acute respiratory distress syndrome. Summary of the experimental evidence for the clinical practice guideline. Ann Am Thorac Soc 14:S261-S270 